UCRL-JC-126841

PREPRINT

\title{
Laser Welding and Collagen Crosslinks
}

\author{
K. M. Reiser, W. Small, D. J. Maitland, \\ N. J. Heredia, L. B. Da Silva, D. L. Matthews, \\ and J. A. Last
}

This paper was prepared for submittal to the Society of Photo-Optical Instrumentation Engineers International

Symposium on Optical Applied Science and Engineering

San Jose, California

February 8-14, 1997

February 20, 1997

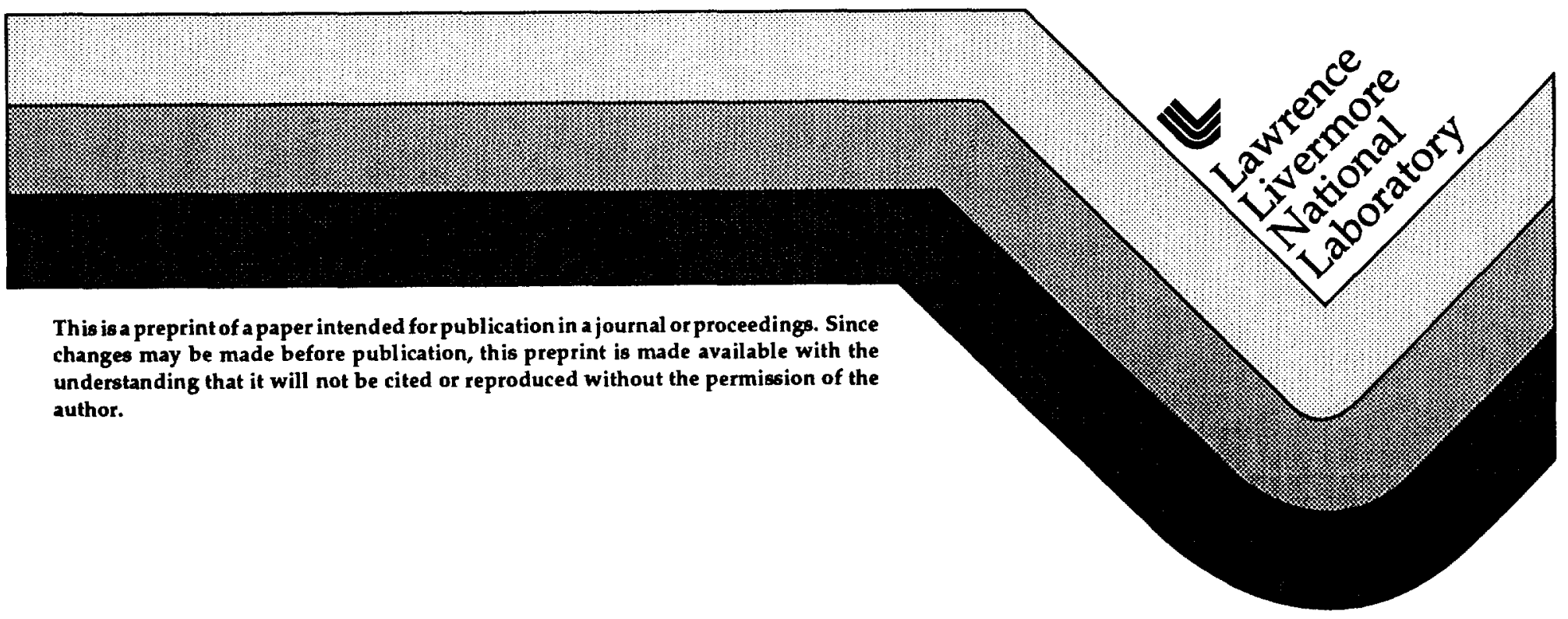




\section{DISCLAIMER}

This document was prepared as an account of work sponsored by an agency of the United States Government. Neither the United States Government nor the University of California nor any of their employees, makes any warranty, express or implied, or assumes any legal liability or responsibility for the accuracy, completeness, or usefulness of any information, apparatus, product, or process disclosed, or represents that its use would not infringe privately owned rights. Reference herein to any specific commercial product, process, or service by trade name, trademark, manufacturer, or otherwise, does not necessarily constitute or imply its endorsement, recommendation, or favoring by the United States Government or the University of California. The views and opinions of authors expressed herein do not necessarily state or reflect those of the United States Government or the University of California, and shall not be used for advertising or product endorsement purposes. 


\title{
Laser welding and collagen crosslinks
}

\author{
Karen M. Reisera, Ward Small IVb, Duncan J. Maitland ${ }^{b}$, Nicholas J. Herediab, \\ Luiz B. Da Silva ${ }^{b}$, Dennis L. Matthews ${ }^{b}$, Jerold A. Last ${ }^{a}$ \\ aDept. of Medicine, School of Medicine, University of California, Davis, CA 95616 \\ bLawrence Livermore National Laboratory, L-399, P.O. Box 808, Livermore, CA 94550
}

\begin{abstract}
The strength and stability of laser-welded tissue may be influenced, in part, by the effects of laser exposure on collagen crosslinking. We therefore studied the effects of diode laser exposure (805 nm , 1-8 watts, 30 seconds) + indocyanine green dye (ICG) on calf tail tendon collagen crosslinks. The effect of ICG dye alone on crosslink content prior to laser exposure was investigated; unexpectedly, we found that ICG-treated tissue had significantly increased DHLNL and OHP, but not HLNL. Laser exposure after ICG application reduced elevated DHLNL and OHP crosslink content down to their native levels. The monohydroxylated crosslink HLNL was inversely correlated with laser output ( $p<0.01$ by linear regression analysis). DHLNL content was highly correlated with content of its maturational product, OHP, suggesting that precursor-product relationships are maintained.
\end{abstract}

We conclude that: (1) ICG alone induces DHLNL and OHP crosslink formation; (2) subsequent laser exposure reduces the ICG-induced crosslinks down to native levels; (3) excessive diode laser exposure destroys normally occurring HLNL crosslinks.

\section{INTRODUCTION}

Although laser welding offers many potential advantages over conventional tissue closure methods, there is little consensus as to how best to use the rapidly proliferating array of available laser systems. There is even less consensus as to how to develop successful welding protocols. Some of these difficulties result from our ignorance of the molecular mechanisms responsible for laser-mediated tissue bonding, and of the interplay between laser-induced changes in tissue structure and the course of wound healing. In addition, evolution of laser welding techniques has been largely an empirical process, driven more by technological advancement than by theoretical insights. As there is little consensus as to the most appropriate parameters for assessing "goodness of weld", it is difficult to compare results from different groups, or to interpret discrepant results. However, as underlying mechanisms are elucidated, the gap between technology and theory may lessen. Eventually, as laser welding is increasingly viewed as a component of wound healing rather than an isolated event, "goodness of weld" will come to have a commonly accepted meaning.

In the present study, we address basic questions concerning effects of indocyanine green dye (ICG) mediated diode laser welding on collagen structure at the molecular level, specifically, the effects on lysine-derived crosslinks. These crosslinks comprise a family of covalent bonds that not only link two or more collagen molecules together, but also stabilize the spatial relationships among the molecules, so that they form a highly ordered array. The number and type of the collagen crosslinks present in a given tissue are the major determinants of the functional properties of that tissue. Thus, immediate post-weld strength as well as longterm stability of laser welded tissue may depend, in large part, on the effects of laser welding on collagen crosslinks already present in tissue, as well as on crosslinking synthesized as part of the wound healing process. If a laser welding procedure generates widespread perturbations in the crosslinking process, either acutely or chronically, there may be long-term adverse effects, which could range from failure to heal and inadequate wound strength on the one hand, to hypertrophic scarring and fibrosis on the other. To address this issue systematically, we have developed an in vitro system for exposing tissue to a defined ICG mediated diode laser welding protocol. We report herein on the effects on specific crosslinks, and the implications of these findings for refinement of laser welding protocols.

This work was performed under the auspices of the U.S.Department of Energy by Lawrence Livermore National Laboratory under contract no. W-7405-Eng-48. 


\section{MATERIALS AND METHODS}

\subsection{Tissue source}

Tail tendons were obtained from calves aged 3-5 months. Tendon fascicles $5-8 \mathrm{~cm}$ in length were dissected out and stored frozen in normal saline until exposed to laser.

\subsection{Laser exposure}

Tendon fascicles $25 \mathrm{~mm}$ in length were placed on a glass cell. Each specimen was soaked in a solution of $0.25 \%$ weight-by-volume ICG for $\mathbf{4 0} \mathrm{min}$ before laser exposure. Laser treatments were performed with a diode laser at $805 \mathrm{~nm}$. Output power was measured with a power meter. Tissues were exposed to 1, 2, 4, or 8 watts of power. The laser spot ( $2 \mathrm{~mm}$ diameter) was scanned at constant velocity along the entire length of the tendon; average duration of exposure was 30 seconds from end-to-end. Samples were immediately frozen following laser treatment until biochemical analysis. One set of samples was soaked in ICG for $40 \mathrm{~min}$, but not exposed to laser light.

\subsection{Crosslink analyses}

For biochemical analysis, each tendon fascicle was sliced into segments $2-3 \mathrm{~mm}$ long. The number of segments per fascicle ranged from 3 to 5 . Each segment was analyzed individually for collagen crosslink content, using previously described techniques ${ }^{1}$. First, the samples were washed overnight in $5 \mathrm{mM}$ phosphate buffer containing $0.9 \%$ sodium chloride $(\mathrm{NaCl}), \mathrm{pH} 7.4$. The next day, the wash fluid was

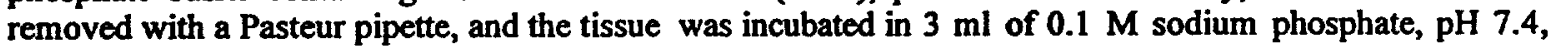
for four hours at room temperature (about $25^{\circ} \mathrm{C}$ ). Tritiated sodium borohydride (142 Ci/Mol) was then added at a ratio of one part per 30 parts (dry weight) of the sample. After one hour the reduction was stopped by the addition of about $1 \mathrm{ml}$ of glacial acetic acid (to $\mathrm{pH} 3$ to 4). The tissues were then thoroughly rinsed with distilled water, hydrolyzed in $\mathrm{N}$ hydrochloric acid $(\mathrm{HCl})$ for 18 hours at $110^{\circ} \mathrm{C}$, rotary evaporated to remove $\mathrm{HCl}$, and filtered using a Rainin microfiltration apparatus. Hydroxyproline content of the hydrolysates was determined by a colorimetric assay 2 .

Crosslinks were analyzed using high performance liquid chromatography methods described by us in detail previously ${ }^{1}$. For DHLNL and HLNL analysis, aliquots of the hydrolysate containing $50 \mathrm{mg}$ of hydroxyproline were chromatographed on a $\mathrm{C}_{18}$ reverse-phase column (Ultrasphere $0.4 \times 25 \mathrm{~cm}$ ). An. isocratic elution system was used; the buffer consists of $22.5 \% \mathrm{n}$-propanol in $0.1 \mathrm{M}$ phosphate buffer, pH 2.83 , containing $0.3 \%$ sodium dodecylsulfate. The flow rate was $0.8 \mathrm{ml} / \mathrm{min}$. Amino acids and difunctional crosslinks in the effluent were visualized by their fluorescence (excitation filter cutoff $=360 \mathrm{~nm}$, emission filter cutoff $=455 \mathrm{~nm}$ ), using a Gilson Spectra-Glo Photometer, after postcolumn derivatization with $\mathbf{0}-$ pthalaldehyde (OPA). In this system the difunctional crosslinks were completely separated from each other and were eluted well after arginine so they can be directly visualized even in an unfractionated hydrolysate. Fractions $(1.3 \mathrm{ml})$ were collected from the fluorometer effluent every minute for determination of radioactivity by liquid scintillation counting. Samples were counted in $8 \mathrm{ml}$ of scintillation fluid at an efficiency of about $33 \%$. Every hydrolysate was run in duplicate; variance between runs was $5 \%$ or less.

Samples were analyzed for the nonreducible trifunctional fluorescent crosslink hydroxypyridinium OHP; its deoxy analogue, lysyl pyridinium, was not detectable. Samples containing 5 ug of hydroxyproline were chromatographed on a $0.4 \times 25 \mathrm{~cm}$ C18 reverse phase column (Ultrasphere, Altex, Berkeley, CA), with an elution solvent of $18 \%$ acetonitrile in $0.01 \mathrm{M}$ heptafluorobutyric acid. A Hitachi 2000 fluorometer was used for detection (excitation $=295 \mathrm{~nm}$, emission=395 nm). Duplicate runs were performed for each hydrolysate; again, variance was less than $5 \%$.

\section{RESULTS}

\subsection{Effects of $1 C G$ and diode laser exposure on crosslinks}

In normal calf tail tendon HLNL is the predominant difunctional crosslink; the mean HLNL content was $0.25 \mathrm{~mol}$ per mol of collagen, consistent with other literature reports. DHLNL was detectable, but at much 
lower concentrations, with mean concentration approximately $0.01 \mathrm{~mol}$ per mol of collagen. This finding is consistent with the literature: in most fibrillar tissues DHLNL decreases rapidly during the postnatal period relative to HLNL. The maturation product of DHLNL, the trifunctional crosslink OHP, was similarly low, with content also approximately $0.01 \mathrm{~mol}$ per $\mathrm{mol}$ of collagen.

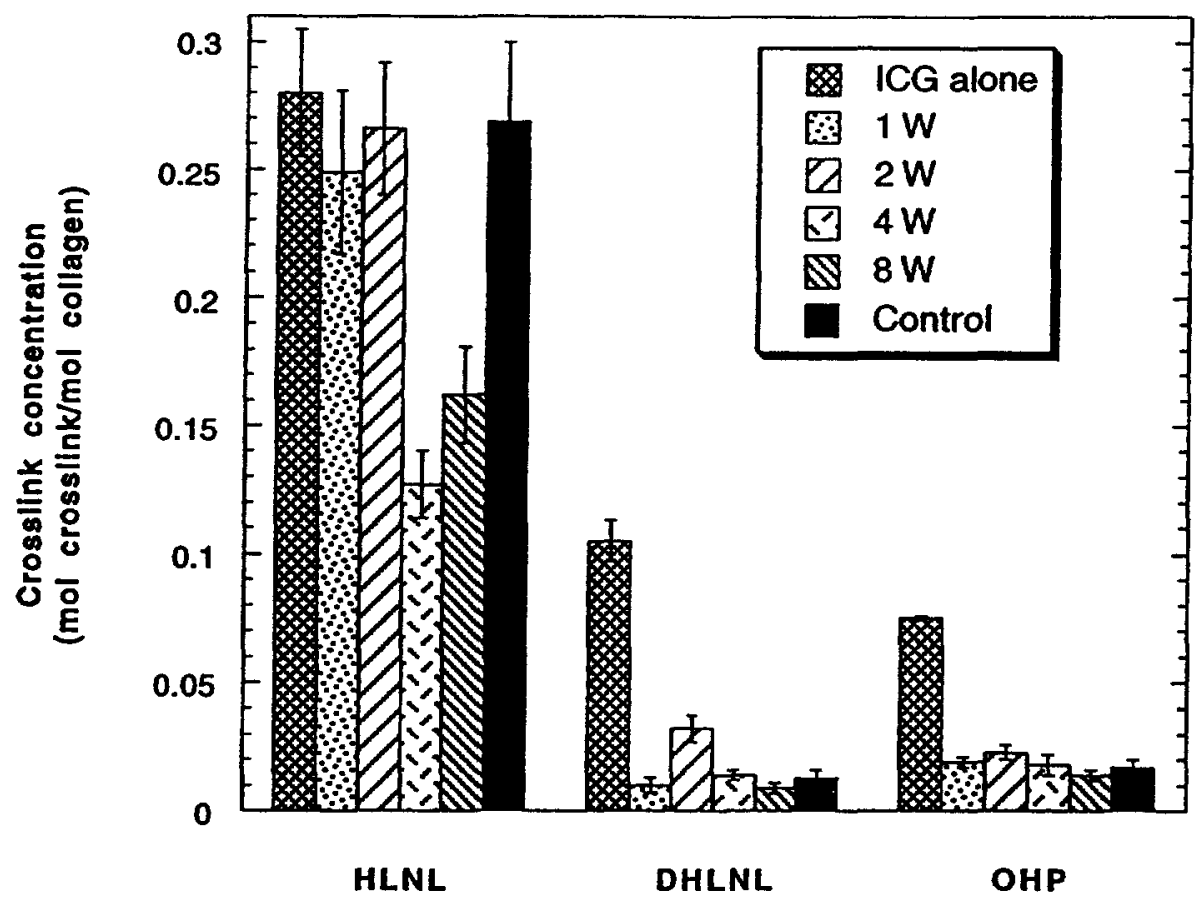

Fig. 1. Effect of ICG and subsequent diode laser exposure on collagen crosslinks. Diode laser powers of $1,2,4$, and $8 \mathrm{~W}$ were used.

Because ICG potentially could react with collagen, we investigated the effects of applying dye to tendon without subsequent laser exposure. Results were surprising: we found that ICG-treated tissue had significantly elevated values for DHLNL and OHP relative to control tissue. Values for HLNL, however, were not significantly different from control values (Fig. 1). To confirm that the apparent increase in OHP was in fact due to OHP, and not to a spurious fluorophore generated by dye exposure, we placed the sample in a UV lightbox overnight; rechromatography showed absence of fluorescence at the OHP elution position, indicating that the increased size was completely attributable to OHP (since it was degraded by UV exposure).

Laser exposure was associated with variable effects on crosslink content, depending on power applied and specific crosslink. DHLNL and OHP were reduced from their ICG-induced elevated values down to native levels. HLNL content was decreased with respect to native levels in tendons exposed to laser at 4 or 8 watts. These data are summarized in Fig. 1. We found that there was a significant inverse relationship between HLNL content and laser power output. Linear regression analysis showed a slope of -0.46 , with $\mathrm{p}<0.01$.

\subsection{Intrasample variability in response to laser exposure}

Our experimental design was aimed at determining if tissue response to laser exposure was uniform over the surface of the exposed tissue with respect to crosslink content. Fascicles were therefore sliced crosssectionally into lengths of $2-3 \mathrm{~mm}$, and each section was analyzed individually. Thus, by averaging results from all the sections we were still able to determine the mean effect on the tendon as a whole. We found that variance among values for crosslinks in individual sections was not appreciably greater in laser-exposed tissues as compared with controls. 


\subsection{DHLNL and OHP correlation}

The relationship between the precursor DHLNL and its product OHP was investigated for treated and control tendons. The results are shown in Fig. 2.

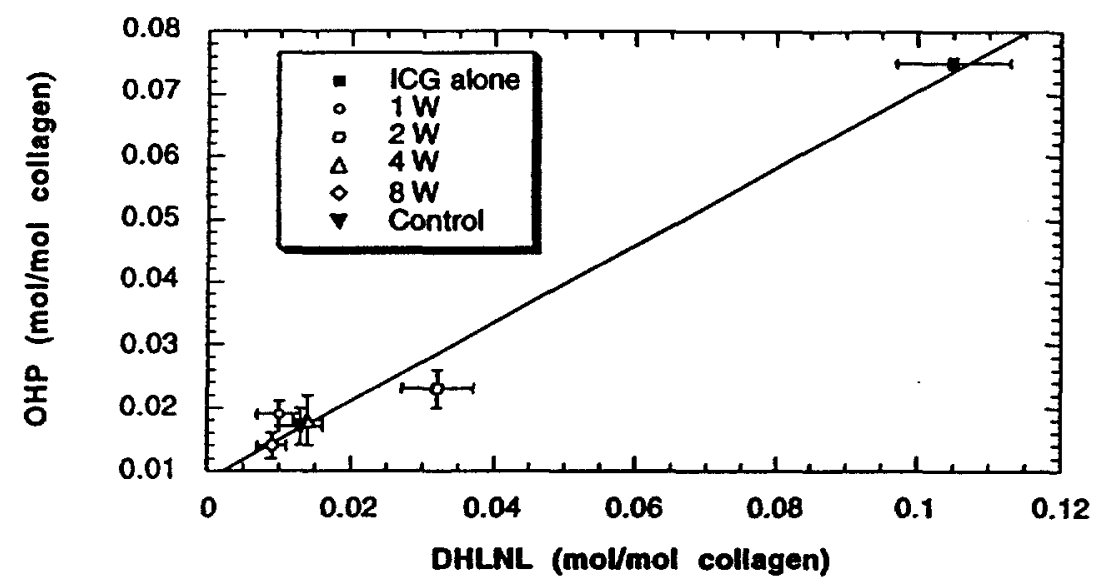

Fig. 2. Relationship between DHLNL content and OHP content. Linear regression analysis was used to investigate the relationship between the precursor DHLNL and its product OHP in treated and control tendons. There was a highly significant correlation between DHLNL content and OHP content.

\section{CONCLUSIONS}

In summary, we have developed a system for exposing tissue to laser light of defined power output. We found that crosslink content was affected by laser exposure, and that laser power output was a major determinant of laser effects on at least one crosslink, HLNL. Our results differ from those reported by Bass et al. ${ }^{3}$, who also looked at the effect of diode laser + ICG exposure on type I collagen. They inferred from their structural data that all covalent crosslinking is destroyed by laser exposure. Further, we determined that ICG alone, without laser exposure, induces the formation of DHLNL and OHP crosslinks. Also, we found that the relationship between the precursor DHLNL and its product OHP is maintained in treated tissues.

From our study, we conclude that: (1) application of ICG prior to diode laser welding may reduce the number of crosslinking sites available for tissue-to-tissue fusion; (2) subsequent diode laser exposure may reduce the elevated DHLNL and OHP levels induced by ICG down to their native levels; (3) excessive laser heating may destroy normally occurring HLNL crosslinks.

\section{ACKNOWLEDGMENTS}

This work was supported by Intra-university Memorandum Agreement B291526 between Lawrence Livermore National Laboratory and UC Davis.

\section{REFERENCES}

1. Buckingham B, Reiser KM. "Relationship between the content of lysyl oxidase-dependent crosslinks in skin collagen, nonenzymatic glycosylation and long-term complications in type I diabetes mellitus", $J$. Clin. Imvest. 86:1046-1054, 1990.

2. Woessner JF. "The determination of hydroxyproline in tissue and protein samples containing small proportions of this imino acid", Arch. Biochèm. Biophys. 93:440-447, 1961.

3. Bass LS, Moazami N, Pocsidio J, Oz MC, LoGerfo P, Treat MR. "Changes in type I collagen following laser welding", Lasers in Surgery and Medicine 12:500-505, 1992. 


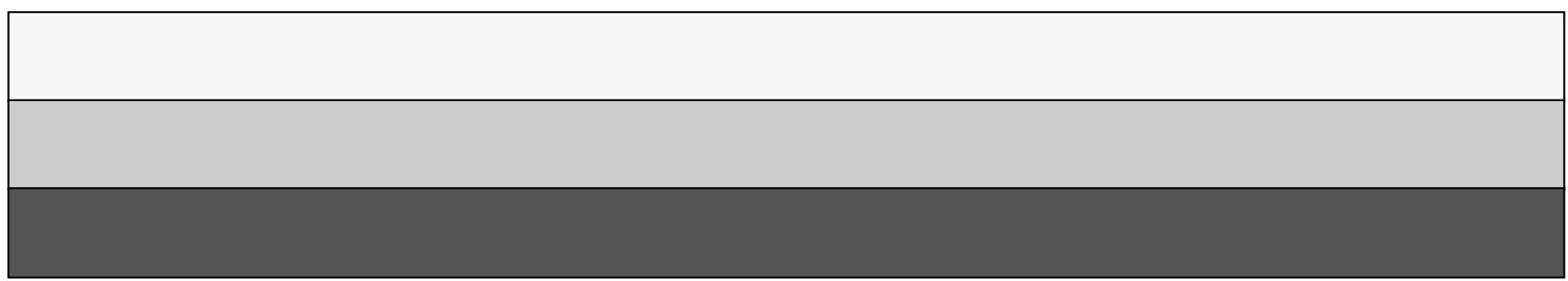

